\title{
MODELING SOCIAL GAUSS-MARKOV MOBILITY FOR OPPORTUNISTIC NETWORK
}

\author{
GuoDong Kang ${ }^{1}$ Jinzhi Ning $^{1}$ and GuoLiang Kang ${ }^{2}$ \\ ${ }^{1}$ DFH Satellite Co., Ltd., 100094, Beijing, China \\ kongton $584 @ 163$. com \\ ${ }^{2}$ University of Technology, Sydney 15 Broadway, Ultimo NSW 2007 \\ kgl.prml@gmail.com
}

\begin{abstract}
Mobility is attracting more and more interests due to its importance for data forwarding mechanisms in many networks such as mobile opportunistic network. In everyday life mobile nodes are often carried by human. Thus, mobile nodes' mobility pattern is inevitable affected by human social character. This paper presents a novel mobility model (HNGM) which combines social character and Gauss-Markov process together. The performance analysis on this mobility model is given and one famous and widely used mobility model (RWP) is chosen to make comparison..
\end{abstract}

\section{KEYWORDS}

Mobility, Social, Gauss-markov, Opportunistic

\section{INTRODUCTION}

With rapid growing popularity of small portable devices (e.g., smart phones, PDAs, laptops) in personal communication domain, a prospective networking scenario occurs where a large number of mobile nodes share or forward information by means of opportunistic encounter[16]. Since these mobile nodes are carried by human, modeling mobility based on realistic human mobile pattern is becoming an essential and important thing. Normally people live in various social circles such as their family circle, their friends circle and so on. This kind of social property influences human movement pattern and further affects nodes' pattern as well. Sometimes in a social circle some people are more popular than others and have more chance to encounter others. The nodes carried by this kind of person can be regarded as one suitable choice for relaying data with other social circles. In other words, these persons could be selected as heads of the corresponding circles and the nodes carried by them could be regarded as one head node. To construct this kind of social opportunistic mobility, this paper adopts random graph for setting up social relations and Gauss-Markov theory for solving individual mobile pattern. 
The rest of this paper is organized as follows: Section II describes the allocation method of mobile nodes. Section III proposes the mathematical model of the mobility. Section IV gives analysis of the proposed mobility model. Conclusion is given in Section V.

\section{GROUP ALLOCATION METHOD}

People's social circle character has a significant impact on nodes mobility pattern. Nodes in different social circles can be allocated into different groups. Node often has more relations with other nodes inside the same group or circle than with those nodes belonging to different groups or circles.

\subsection{Node Relation Setup}

Normally, there is some kind of social or biological relations among those nodes since they are carried by people. To set up this kind of mutual relation, we adopt the classical method of representing social or biological network, weighted graphs. The strength of mutual relation between any node pair is represented using a value in the range $[0,1]$. As a consequence, the network internal relation can be described as a relation matrix with a dimension of $\mathrm{N}^{*} \mathrm{~N}$ where $\mathrm{N}$ is the total number of nodes in the network. At present, there are several models that describe the key properties of real-life network, such as random graph, small world, etc. Some research work show that the properties of these random graphs, such as path lengths, clustering coefficients, can't be regarded as accurate models of realistic networks [5, 6, 7]. Here, we choose the geometric random graph to be the network model. In this kind of model, the geometry relations of nodes have strong association with the social relation of nodes. That means when any two nodes are in the radio range of each other, the social relation exists. On the contrary, since they even can't communicate with each other, we think that there is no any social interaction between them. So when the Euclidean distance between any two nodes is smaller than radio range R, the corresponding element of the social relation matrix $M$ is set to be 1 or else set to be 0 as follows.

$$
m_{i, j}=\left\{\begin{array}{l}
1 \text { if } i \neq j \text { and }\left\|P_{i}-P_{j}\right\| \leq R, \\
0 \text { otherwise }
\end{array}\right.
$$

It shall be emphasized that the relations value of one node and itself is regarded to be zero in the matrix. In [6] it is shown that in two or three dimensional area using Euclidean norm can supply surprisingly accurate reproductions of many features of real biological networks. Fig.1 gives one example of 100 nodes.

\subsection{Nodes Group Allocation}

Once the relation matrix $\mathrm{M}$ is obtained, groups can be detected. Group structure is one of the common characters in many real networks. However, finding group structures within an arbitrary network is an acknowledged difficult task. A lot of work has been done on that. Currently, there are several methods that can achieve that goal, such as Minimum-cut method, Hierarchical clustering, Girvan-Newman algorithm, etc. 


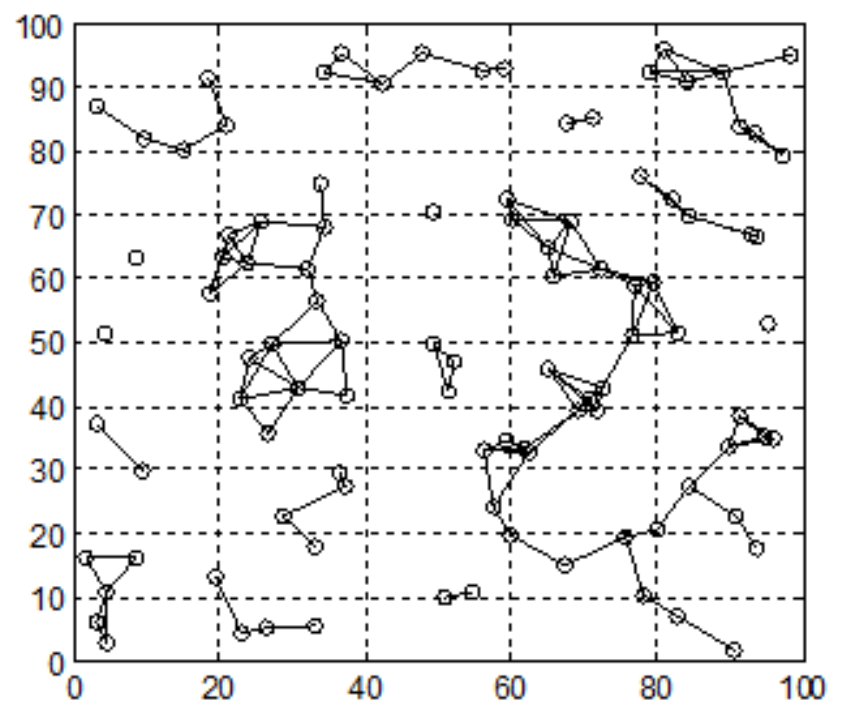

Fig.1. An example of 100 Nodes Relations. (Small circles represent nodes carried by people which are uniformly distributed in one given $100 \mathrm{~m} * 100 \mathrm{~m}$ square; the short straight lines represent the existing relation between node pair.)

Minimum-cut method is one of the oldest algorithms for dividing networks into parts. This method uses in load balancing for parallel computing in order to minimize communication between processor nodes. However, this method always finds communities regardless of whether they are implicit in the structure, and it can only find a fixed number of them. So it is less than ideal for finding community structure in general networks [4].

Hierarchical clustering is another method for finding community structures in network. This method detects the community by defining a similarity measure quantifying some (usually topological) type of similarity between node pairs.

The Girvan-Newman algorithm is one commonly used algorithm for finding communities [12]. It identifies edges in one network that lie between communities and then removes them, leaving behind just the communities themselves. But this method runs slowly which makes it impractical for networks of more than a few thousand nodes [11].

Modularity maximization is one of the most widely used methods for community detection [11]. Modularity is a benefit function that measures the quality of a particular division of a network into communities. This method detects the community structure of high modularity value by exhaustively searching over all possible divisions [8].

In this paper we adopt modularity maximization as the social group detection method. Modularity maximization is one of the most widely used methods for group detection [11].

Modularity is a benefit function that measures the quality of a particular division of a network into groups. This method detects the group structure of high modularity value by exhaustively searching over all possible divisions [8]. 
In real networks, the modularity value is usually in the range [0.3, 0.7]; 1 means a very strong group structure and 0 means no better than random.

\section{MOBILITY MODEL SETUP}

\subsection{Mobility Scenario Description}

One kind of usual mobility scenario appearing in daily life is one people in a group leading the mobility. This person is selected because of his popularity in the group or his familiarity with the environment. He is called as head of the group in this paper. The head moves freely and leads other people to move together. Other people follow head people's direction and adjust their speeds to keep up with him. The head's direction and speed determine the general direction and speed of whole group. Fig.2 gives an illustration of this mobility scenario.

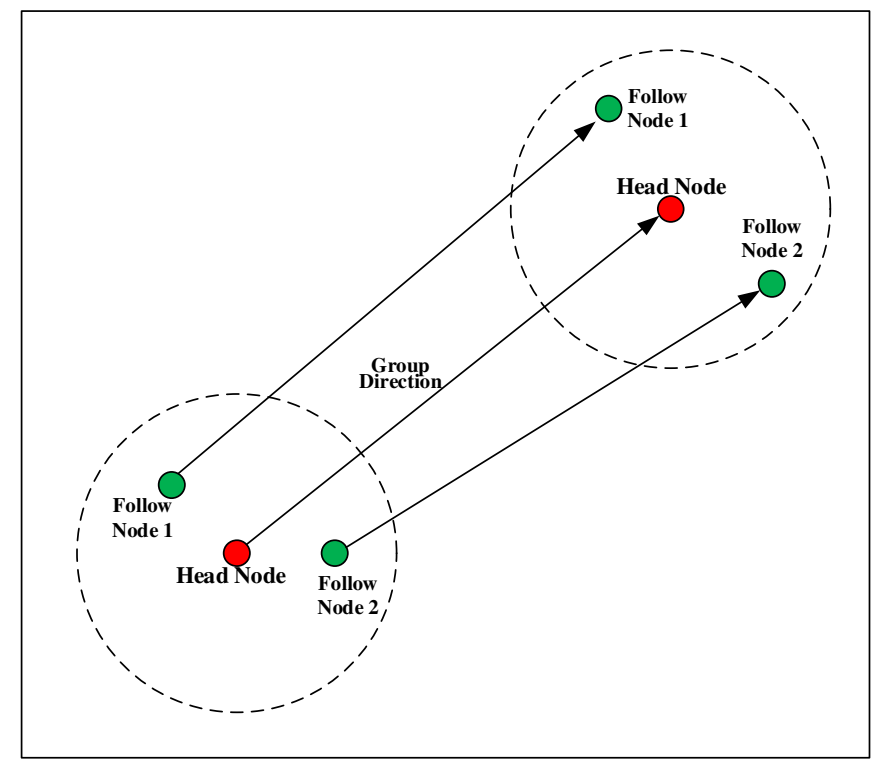

Fig.2. Illustration of mobility scenario. The red solid circle represents head node or head people. The other two blue solid circles represent follow nodes or follow people. The dash circles represent social circles or groups which head node and follow nodes belong to.

\subsection{Mobility Mathematical Model}

Assume there are total $\mathrm{N}$ nodes in one group, the $n^{\text {th }}$ node is denoted as node $n(1 \leq n \leq N)$

\subsubsection{Head Node Determination Scheme}

Assume at instant $t$ there are number $\mathrm{M}$ nodes which react with node $\mathrm{i}$. The following expression describes the average relation value of node $i$ with other nodes :

$$
\bar{m}_{i}(t)=\frac{1}{M} \sum_{j=1}^{N} m_{i, j}(t) \quad(i \neq j)
$$


where

$N \quad$ is the total number of the network;

$M \quad$ is the number of nodes in the network characterized by $m_{i, j}>0$

$\bar{m}_{i}(t)$ is the average relation value of node $\mathrm{i}$ at instant $\mathrm{t}$;

$m_{i, j}(t)$ is the element of the relation matrix at instant $\mathrm{t}$;

In this paper, the node which has the greatest average relation values will be assigned to be the Head node of the group and the remaining nodes in the group will be regarded as Follow nodes which will follow the head node. At the beginning, the head node of the group is denoted as node $\mathrm{h}$, it has

$$
\bar{m}_{h}(t \mid t=0) \geq \bar{m}_{i}(t \mid t=0) \quad(1 \leq i \leq N, i \neq h)
$$

where $\bar{m}_{h}(t)$ is the average relation value of node $\mathrm{h}$ at time $\mathrm{t}$;

Initially, the head node is chosen as the node which is most popular in the group, namely has the strongest relations with other member nodes of the group. However, as the time past, the mutual relation between the initial assigned head node and other nodes in the group may vary. Maybe at time $t_{e}$ the initial head node don't have the strongest relation with others any more. Consequently, one election shall take place to determine the next new head node who has the strongest relation with other nodes at time $t_{e}$. The time $t_{e}$ is called head node determination period in this paper. The average relation value of the new elected head node, $h_{E}$, has

$$
\bar{m}_{h_{E}}\left(t \mid t=t_{e} \bullet E\right) \geq \bar{m}_{i}\left(t \mid t=t_{e} \bullet E\right) \quad(1 \leq i \leq N, i \neq h)
$$

where

$E \geq 0$ is the head node election number in the group;

$t_{e} \quad$ is the head node election period.

\subsubsection{Gauss-Markov process setup}

A Gauss-Markov process is a simple stochastic process with many social applications. In terms of continuous time, a stationary Gauss-Markov process can be described by the following autocorrelation function $[16,18]$ :

$$
R_{f}(\tau)=E[f(t) f(t+\tau)]=\sigma^{2} e^{-\beta|\tau|}
$$

where

$\sigma^{2} \quad$ is the variance;

$\beta \geq 0$ determine the degree of memory in the process.

Assume to discretize time into serial time slots $\Delta t$ (normalized to 1 throughout this paper),

$$
f(t)=f(s \cdot \Delta t)
$$


Define $\alpha=e^{-\beta \Delta t}$, then the discrete representation of (1) is

$$
f(s \cdot \Delta t)=\alpha f[(s-1) \cdot \Delta t]+(1-\alpha) \mu+\sqrt{1-\alpha^{2}} d_{\text {Gauss }}
$$

where

$$
\begin{aligned}
& 0 \leq \alpha \leq 1 ; \\
& \mu \quad \text { is the asymptotic mean of } f \text { when s approach infinity. }
\end{aligned}
$$

$d_{\text {Gauss }}$ is an independent, uncorrelated, and stationary Gaussian process, with mean $\mu_{\text {Gauss }}$ equals to zero and standard deviation $\sigma_{\text {Gauss }}$ equals to $\sigma$ when s approaches infinity.

\subsubsection{Gauss-Markov mobility discussion}

In this paper, nodes' speed and direction are both regarded as Gauss-Markov processes which are correlated with time. Nodes' speed and direction discrete representations of (1) can be described as follows:

$$
\begin{gathered}
V(s \cdot \Delta t)=\alpha V[(s-1) \cdot \Delta t]+(1-\alpha) \mu_{v}+\sqrt{1-\alpha^{2}} d_{\text {Gauss }}^{v} \\
\Phi(s \cdot \Delta t)=\alpha \Phi[(s-1) \cdot \Delta t]+(1-\alpha) \mu_{\phi}+\sqrt{1-\alpha^{2}} d_{\text {Gauss }}^{\phi}
\end{gathered}
$$

Gauss-Markov allows previous speed or direction to influence current speed or direction. Moreover, Gauss-Markov is a memory-tunable process accounting for its suitableness for the description of relations of follow nodes and head node about speed and direction choices. The parameter, $\alpha$, is tunable which determines the amount of memory and variability in movement. The following discusses three cases of parameter $\alpha$.

Case 1: $\alpha=0$

When $\alpha=0$, Gauss-Markov becomes memoryless; The speed and direction of nodes completely depend on their average $\mu_{v}, \mu_{\phi}$ and the Gauss variables.

$$
\begin{aligned}
& V(s \cdot \Delta t)=\mu_{v}+d_{\text {Gauss }}^{v} \\
& \Phi(s \cdot \Delta t)=\mu_{\phi}+d_{\text {Gauss }}^{\phi}
\end{aligned}
$$

This paper thinks this case is quite suitable for setting up the relations of speed and direction between follow nodes and head node by setting $\mu_{f n v}, \mu_{f u \phi}$ of follow nodes to identify $\mu_{h v}, \mu_{h \phi}$ of head node since follow nodes have one common character that is they always try to keep up with head node movement.

Case 2: $\alpha=1$

When $\alpha=1$, Gauss-Markov loses all the variables becoming predictable pattern. The current speed and direction is totally identical to the previous values. The movement becomes a straight line. 


$$
\begin{gathered}
V(s \cdot \Delta t)=V[(s-1) \cdot \Delta t] \\
\Phi(s \cdot \Delta t)=\Phi[(s-1) \cdot \Delta t]
\end{gathered}
$$

This paper thinks this case is suitable for head node mobility since his destination is explicit.

Case 3: $0<\alpha<1$

This is intermediate randomness case between the two above cases. This case won't be taken into account too much here.

\subsubsection{Head node mobility setup}

Normally, the head node in a group shall know its destination explicitly. Once he determines the destination, he will move straightly toward the destination maintaining his speed and direction until he reaches the destination. During head node mobility, he may have several destinations thus has several corresponding mobility sections. Each mobility section is one $\alpha=1$ case GaussMarkov process. The speed and direction of different mobility sections are different. Head node mobility is set up as follows.

Assume at instant $t_{i}$ one destination of head node $h$ is chosen and at instance $t_{j}$ the head node reaches this destination. The destination of head node can be denoted as

$$
P_{d h}\left(t \mid t_{i} \leq t \leq t_{j}\right)=\left[X_{d h}\left(t_{i}\right) \quad Y_{d h}\left(t_{i}\right)\right]
$$

Normalize $\Delta t$ to be 1 , at instant $\mathrm{t}\left(t_{i}<t \leq t_{j}, t=s \cdot \Delta t\right)$, the position of node $h$ is a function of coordinate $X_{h}(t)$ and $Y_{h}(t)$ which can be expressed as follows:

$$
P_{h}(s \cdot \Delta t)=\left[\begin{array}{c}
X_{h}(s \cdot \Delta t) \\
Y_{h}(s \cdot \Delta t)
\end{array}\right]=\left[\begin{array}{c}
X_{h}[(s-1) \cdot \Delta t]+V_{h}(s \cdot \Delta t) \cdot \cos \Phi_{h}(s \cdot \Delta t) \\
Y_{h}[(s-1) \cdot \Delta t]+V_{h}(s \cdot \Delta t) \cdot \sin \Phi_{h}(s \cdot \Delta t)
\end{array}\right]
$$

where

$s \quad$ is the number of $\Delta t$ at current instant;

$s-1$ is the number of $\Delta t$ at previous instant;

$\Phi_{h} \quad$ is the direction function of node $\mathrm{h}$ which can be defined as

$$
\Phi_{h}(t)=\operatorname{arctg}\left(\frac{\left|Y_{d h}\left(t_{i}\right)-Y_{h}\left(t_{i}\right)\right|}{\left|X_{d h}\left(t_{i}\right)-X_{h}\left(t_{i}\right)\right|}\right)
$$

$V_{h}$ is the speed function obeying normal distribution which is determined with destination synchronously.

The research in [13] has shown that the walking speed of a human obeys normal distribution. The measurement in [14] shows that the mean speed of a walking human is a range which is from $1.16 \mathrm{~m} / \mathrm{s}$ to $1.58 \mathrm{~m} / \mathrm{s}$ representing walking normally or walking fast. In [15], the Manual of Uniform Traffic Control Devices (MUTCD) shows that human walk with a normal speed of 1.2 $\mathrm{m} / \mathrm{s}$ (4 ft/sec). [10] indicates a statistics that walking speed for younger human (ages 14 to 64) 
was $1.25 \mathrm{~m} / \mathrm{sec}(4.09 \mathrm{ft} / \mathrm{sec})$; for older human (ages 65 and over) it was $0.97 \mathrm{~m} / \mathrm{sec}(3.19 \mathrm{ft} / \mathrm{sec})$. For designing purposes values of $1.22 \mathrm{~m} / \mathrm{sec}(4 \mathrm{ft} / \mathrm{sec})$ for younger human and $0.91 \mathrm{~m} / \mathrm{sec}(3$ $\mathrm{ft} / \mathrm{sec}$ ) for older human are appropriate [10]. In this paper, the mean value of walking speed of the head node, $\mu_{h v}$, equals to $1.22 \mathrm{~m} / \mathrm{s}$ [10] and its standard deviation $\sigma_{h v}$ is $0.26 \mathrm{~m} / \mathrm{s}[13]$. So the head node's speed at time $t$ is

$$
V_{h}(t)=V_{h}\left(t_{i}\right) \sim N\left(\mu_{h v}, \sigma_{h v}\right)
$$

The probability density function of $V_{h}(t)$ can be expressed as:

$$
f(v)=\frac{\exp \left(-\frac{\left(v-\mu_{h v}\right)^{2}}{2 \sigma_{h v}^{2}}\right)}{\sigma_{h v} \sqrt{2 \pi}}
$$

Once head node reaches the destination, he will go towards his next destination which is uniformly chosen in given movement area.

\subsubsection{Follow nodes mobility setup}

Follow nodes' speed and direction discrete representation of (1) can be described as follows according to case 1:

$$
\begin{aligned}
& V_{f f n}(s \cdot \Delta t)=\mu_{f i v v}+d_{\text {Gauss }}^{v} \\
& \Phi_{f h}(s \cdot \Delta t)=\mu_{f h \phi}+d_{\text {Gauss }}^{\phi}
\end{aligned}
$$

where

$$
\mu_{f n v} \text { equals to } \mu_{h v}
$$

$d_{\text {Gauss }}^{v}$ is an independent, uncorrelated, and stationary Gaussian process, with mean $\mu_{\text {Gauss }}^{v}$ equals to 0 and standard deviation $\sigma_{\text {Gauss }}^{v}$ equals to $\sigma_{h v}$.

Similarly, this paper chooses $\mu_{\text {fun }}=\Phi_{h}$ and $\sigma_{\text {Gauss }}^{\phi}=\pi / 12$.

Consequently, the position of follow node $f n$ is a function of coordinate $X_{f n}(s \cdot \Delta t)$ and $Y_{f n}(s \cdot \Delta t)$ which can be expressed as follows:

$$
P_{f n}(s \cdot \Delta t)=\left[\begin{array}{c}
X_{f n}(s \cdot \Delta t) \\
Y_{f n}(s \cdot \Delta t)
\end{array}\right]=\left[\begin{array}{c}
X_{f n}[(s-1) \cdot \Delta t]+V_{f n}(s \cdot \Delta t) \cdot \cos \Phi_{f n}(s \cdot \Delta t) \\
Y_{f n}[(s-1) \cdot \Delta t]+V_{f n}(s \cdot \Delta t) \cdot \sin \Phi_{f n}(s \cdot \Delta t)
\end{array}\right]
$$

The mean of speed and direction of follow nodes equaling to the values of head node interprets that their mobility shall follow group's movement. The difference of standard deviation of normal distribution between follow-node and head-node reflects these follow-nodes have their freedom degree in mobility to some extent. 


\section{MODEl CHARACTER ANALYSIS}

\subsection{Classical Individual Mobility Model-RWP}

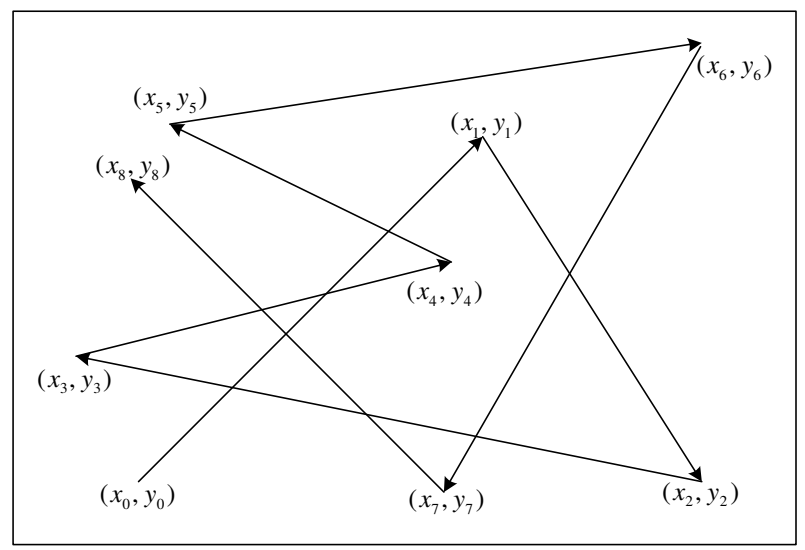

Fig.3. An illustration of RWP

Josh et al. [8] present a Random Waypoint model. In this model a mobile node is initially placed at random location in the simulation area with a destination and speed according to the random distribution. Then the mobile node moves from its current location to its destinations via a straight line. After arrival at the destination, the mobile node determines a pause time according to a random distribution, and after that chooses a new destination.

\subsection{Analysis and Comparision}

To analyze the character of these two mobility models, two important parameters inter-contact time and contact time are adopted. These two parameters describe the characteristics of connection opportunities of the network, i.e. how many and when they occur, how often and how long. Contact time is defined as the time interval during which the two nodes can keep contact. The time interval from this contact to next one is defined as inter-contact time during which nodes can't communicate [1]. Fig.4. gives an illustration. These two parameters are very important for opportunistic network. Contact time can help us determining the capacity of opportunistic networks. Inter-contact time is a parameter which strongly affects the feasibility of the opportunistic networking.

The new mobility model in this paper is named as Head Node Group Mobility Model (HNGM) here. We let each mobility model run 1000 seconds for one experiment and made 50 times similar experiments for each mobility model. Head node election period is set to be 1000 seconds here. Figure 5 and Figure 6 give the contact time distribution and inter-contact time distribution in different coordinate systems. 


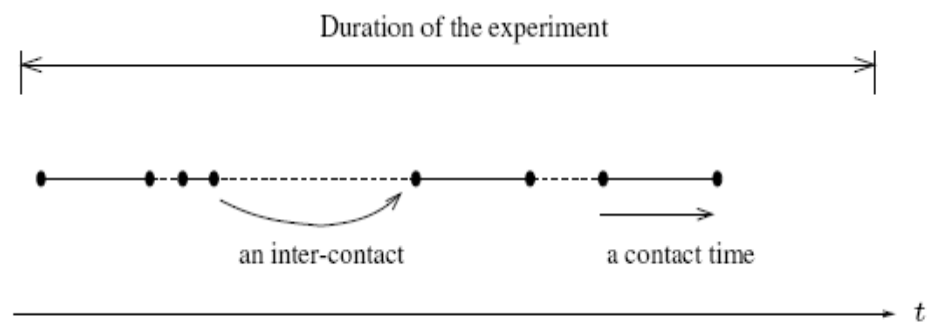

Fig.4. Contact and Inter-contact times for a pair of nodes[1].

In Fig. 5 (a), we can see that the new mobility model' inter-contact time distribution behave an exponential distribution plot using log-log coordinate system. In Fig. 5 (b), the contact time distributions show more evident difference. The RWP mobility models' curves are still exponential-like curves. In contrast, the shape of HNGM model' curves are changed. The HNGM model's curve is one kind of transition from exponential-like curve to power- law-like curve. This shows HNGM model behaves stronger group character than RWP model. In Fig. 6 we can see the difference between the curves more clearly using semi-log coordinate system. The exponential nature of the inter-contact time shown in Fig. 5 (a) becomes straight line form in Fig. 6 (a).

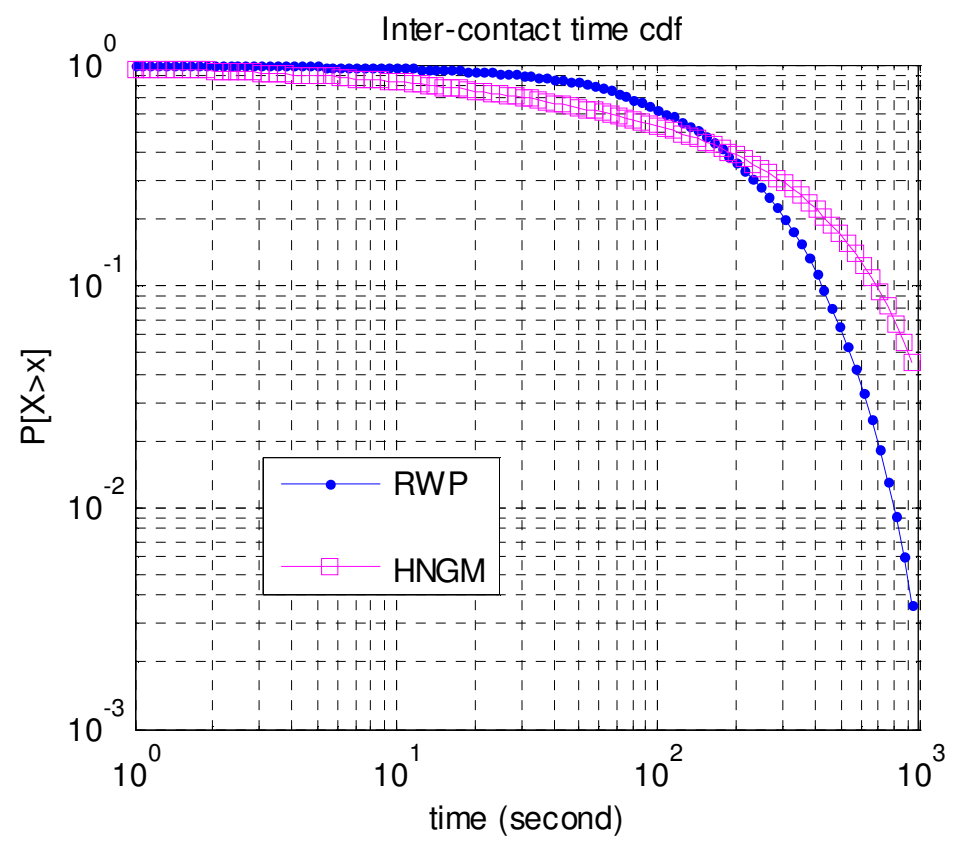

Fig5 (a) 


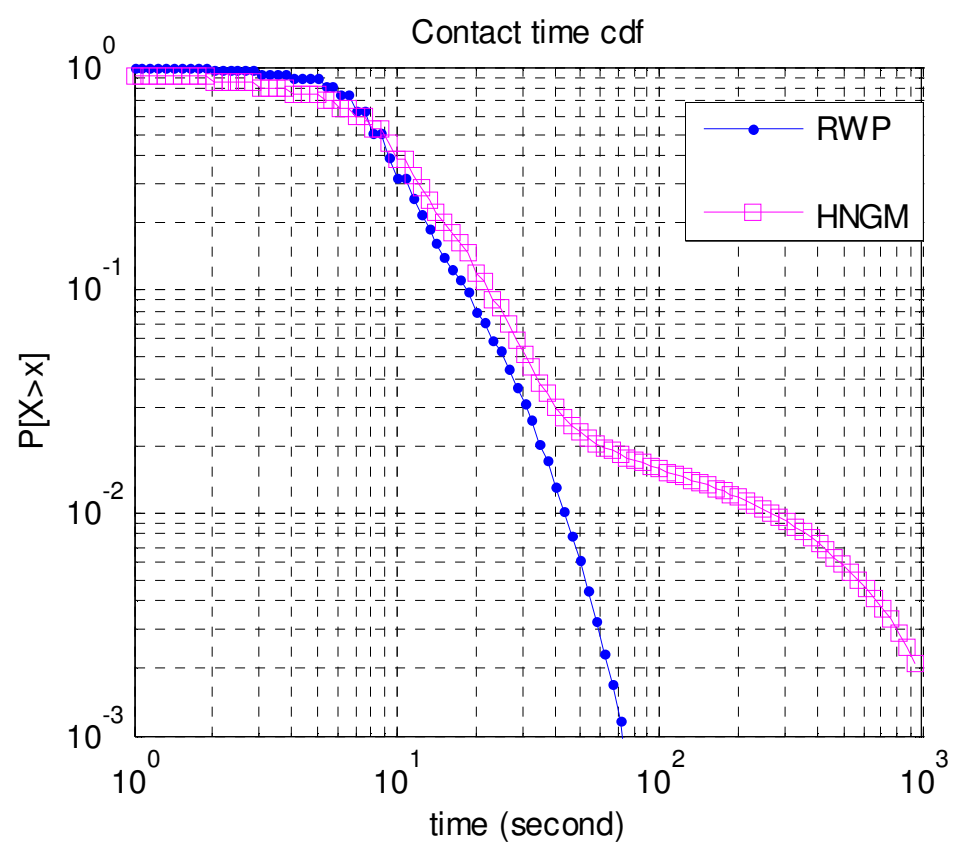

Fig5 (b)

Fig. 5. Inter-contact time cdf and contact time cdf in log-log coordinates

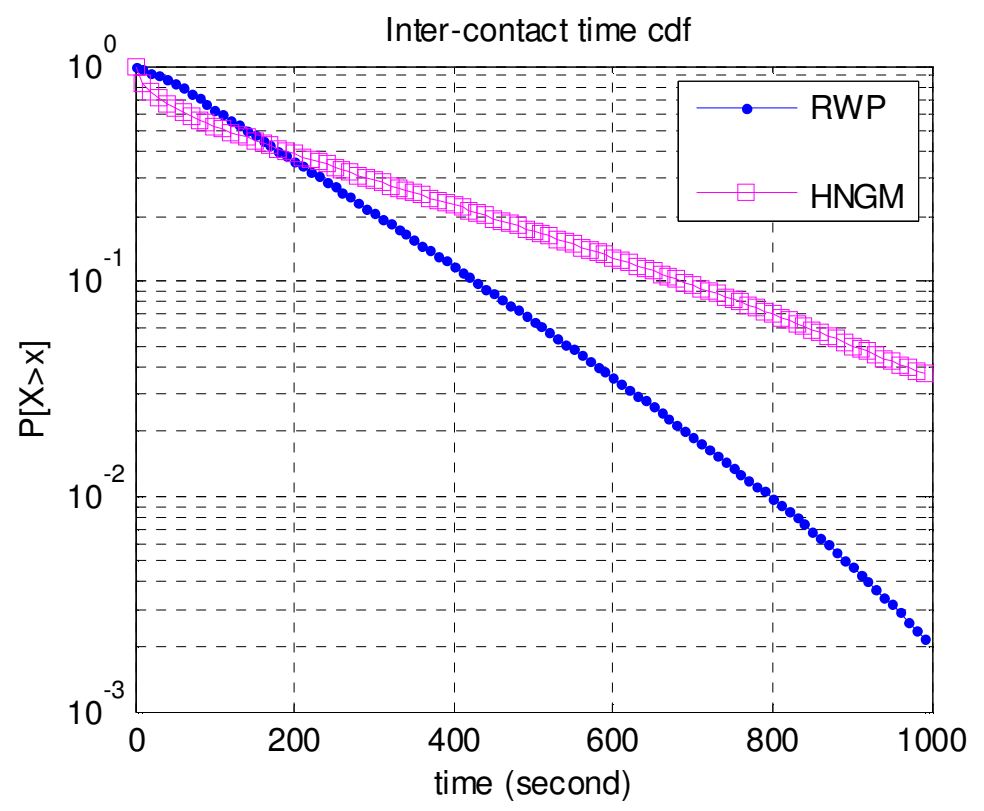

Fig6 (a) 


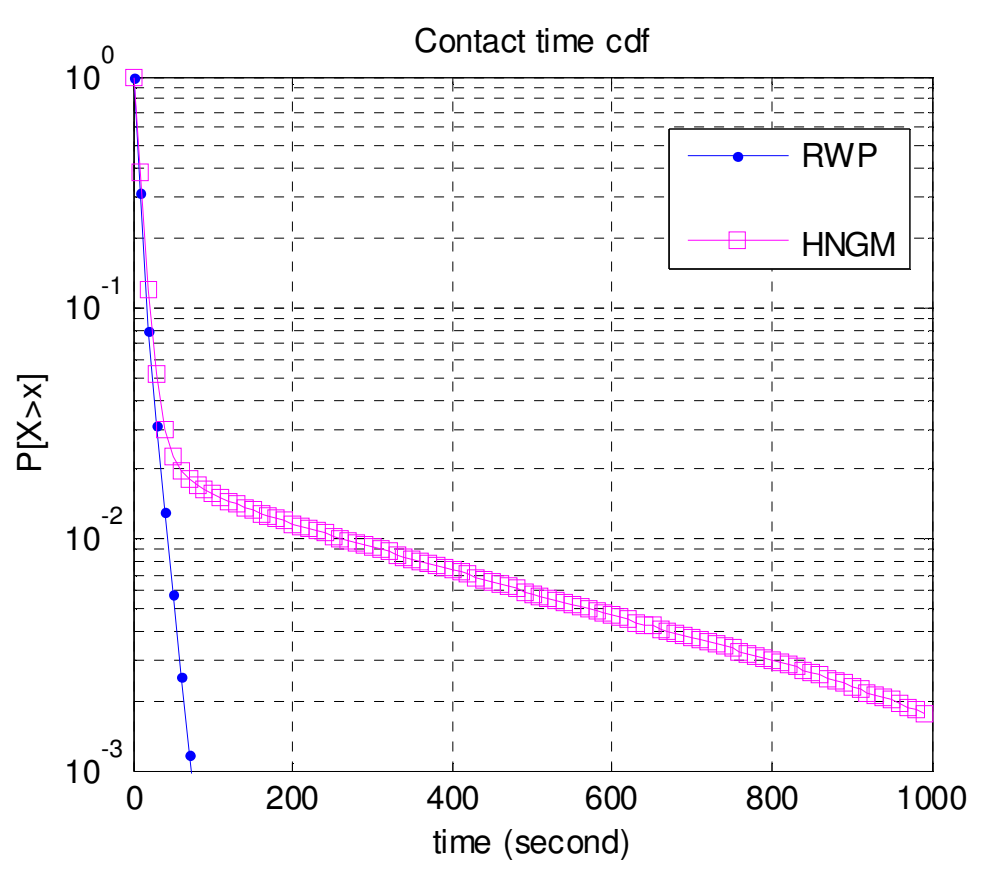

Fig 6 (b)

Fig. 6. Inter-contact time cdf and contact time cdf in semi-log coordinates.

\section{CONCLUSION AND FUTURE WORK}

This paper proposed one new group mobility model using social Gauss-Markov scheme which is based on one social realistic mobility scenario. This paper also analyzes its characters about contact time, inter-contact time. The curves of contact time and inter-contact time show different shapes with the classical individual model RWP. By comparison, HNGM model shows obvious group character than RWP model. In the future work, the author will undertake further research on mobility about $0<\alpha<1$ case of Gauss-Markov process.

\section{ACKNOWLEDGEMENT}

The author would like to thanks DFH Corporation support.

\section{REFERENCES}

[1] A. Chaintreau, P. Hui, J. Crowcroft, C. Diot, R. Gass, and J. Scott, "Pocket Switched Networks: Real-world mobility and its consequences for opportunistic forwarding," Technical Report UCAMCL-TR-617, University of Cambridge, Computer Laboratory, February 2005.

[2] J Broch, DA Maltz, DB Johnson, YC Hu, J Jetcheva," Multi-hop wireless ad hoc network routing protocols", in Proceedings of the ACM/IEEE International Conference on Mobile Computing and Networking (Mobicom 1998), pages 85-97, 1998.

[3] Y. Ko and N.H. Vaidya, "Location-aided routing (LAR) in mobile ad hoc networks," in Proceedings of the ACM/IEEE International Conference on Mobile Computing and Networking (Mobicom), pages 66-75, 1998.

[4] M. E. J. Newman, Detecting community structure in networks. Eur. Phys. J. B, 38, 321-330 (2004).. 
[5] E. de Silva and M. Stumpf, "Complex networks and simple models in Biology", J. R. Soc. Interface, 2 (2005), pp. 419-430..

[6] N. Przulj, D. G. Corneil, and I. Jurisica, "Modeling interactome:Scale- free or geometric?", Bioinformatics, 20 (2004), pp. 3508-3515..

[7] D. J. Watts and S. H. Strogatz, “Collective dynamics of 'small-world' networks”, Nature, 393 (1998), pp. 440-442.

[8] M.E.J. Newman and M. Girvan. "Finding and evaluaing community structure in networks". Physical Review E,68,2003.

[9] Mirco Musolesi, Cecilia Mascolo, "A community based mobility model for ad hoc network research", in Proceedings of the 2nd international Workshop on Multi-Hop Ad Hoc Networks: From theory To Reality (REALMAN '06), Florence, Italy, May 2006, pp 31-38.

[10] R.L. Knoblauch, M.T. Pietrucha, M. Nitzburg. Field studies of pedestrian walking speed and start-up time. Transportation Research Board Records No. 1538, 1996.

[11] Newman, M. E. J. Fast algorithm for detecting community structure in networks. Phys. Rev. E $69,066133(2004)$

[12] Girvan, M. and Newman, M. E. J., Community structure in social and biological networks, Proc. Natl. Acad.Sci. USA 99, 8271-8276 (2002).

[13] L. Henderson. The statistics of crowd fluids. Nature, Vol. no. 229, 381-383, 1971.

[14] Finnis, K.K. and Walton, D. Field observations of factors influencing walking speeds. Ergonomics, 2006.

[15] LaPlante, John and Kaeser, Thomas P. "A History of Pedestrian Signal Walking Speed Assumptions", in Proceedings of the 3rd Urban Street Symposium: Uptown, Downtown, or Small Town: Designing Urban Streets That Work, 2007.

[16] L. Pelusi, A. Passarella, and M. Conti, "Opportunistic networking: data forwarding in disconnected mobile ad hoc networks," Communications Magazine, IEEE, vol. 44, no. 11, pp. 134 -141, november 2006.

[17] B. Liang and Z. Haas. Predictive distance-based mobility management for PCS networks. In Proceedings of the Joint Conference of the IEEE Computer and Communications Societies (INFOCOM), March 1999

[18] E. Wong and B. Hajek, Stochastic Processes in Engineering Systems,Springer-Verlag, 1985

\section{AUTHORS}

GuoDong KANG is an telecommunication engineer of DFH Satellite Co., Ltd., 100094, Beijing, China. 